Supporting Information

\title{
Omniphobic Hollow Fiber Membranes for Water Recovery and Desalination
}

Mahdi Mohammadi Ghaleni, Abdullah Al Balushi, Mona Bavarian, and Siamak Nejati*

*Corresponding author contact: snejati2@unl.edu

Department of Chemical and Biomolecular Engineering

The University of Nebraska-Lincoln, Lincoln, NE 68588-8286, USA 


\section{Section S1. Experimental Section}

\section{Materials and Chemicals}

Polyvinylidene fluoride (PVDF, Kynar ${ }^{\circledR}$ HSV 900) was supplied by Arkema Inc., USA. Triethyl phosphate (TEP, 99.8\%), perfluorobutane sulfonyl fluoride (PBSF, 96\%), and sodium dodecyl sulfate (SDS, 99\%) were purchased from Sigma-Aldrich. Ethanol (99.5\%), isopropyl alcohol (IPA, ACS grade), and sodium chloride ( $\mathrm{NaCl}$, ACS grade) were purchased from Fisher Scientific. Ethylene Glycol (99.5\%) was purchased from ACROS Organics. Hexafluoropropylene oxide (HFPO) was purchased from Oakwood chemical. 1H,1H,2H,2H-perfluorodecyl acrylate (PFDA, 97\%) was obtained from Frontier Scientific. Canola oil was purchased from Wesson Oil. Deionized (DI) water was obtained from a Simplicity® ultrapure water purification system (Millipore, Billerica, MA). Ultrapure nitrogen was purchased from Matheson Gas Company.

\section{Fabrication of PVDF Hollow Fiber Membranes}

Hollow fiber membranes were fabricated using a dry-jet wet-spinning method at room temperature $\left(20{ }^{\circ} \mathrm{C}\right)$ with a relative humidity of $49-52 \%$. Before the spinning experiment, PVDF powder was dried in a vacuum oven at $50{ }^{\circ} \mathrm{C}$. The dope solution was prepared by dissolving the PVDF powder in TEP (12 wt.\%). The mixture was stirred on a hot plate set at $150{ }^{\circ} \mathrm{C}$ for 12 hours. Afterward, the polymer solution was left to cool for four hours, transferred to a stainless-steel syringe, and left overnight to degas. The dope and bore were fed to the spinneret (DeltaE Srl, Italy) using highpressure syringe pumps (Fusion 6000, Chemyx Inc., USA). The fibers were drawn into a 1-Liter cylindrical coagulation bath (NMP/Water, 30/70 wt.\%) to induce nonsolvent induced phase inversion (NIPS); Table S1 includes the detailed spinning parameters. The nascent fibers were left in the coagulation bath for 10 minutes and then transferred to a water bath. After 48 hours, the fibers were moved to an IPA bath and left for another 24 hours to remove excess TEP trapped inside the pores. Finally, the hollow fiber membranes were taped on a glass substrate and dried in the vacuum oven at $50{ }^{\circ} \mathrm{C}$ for 24 hours. 


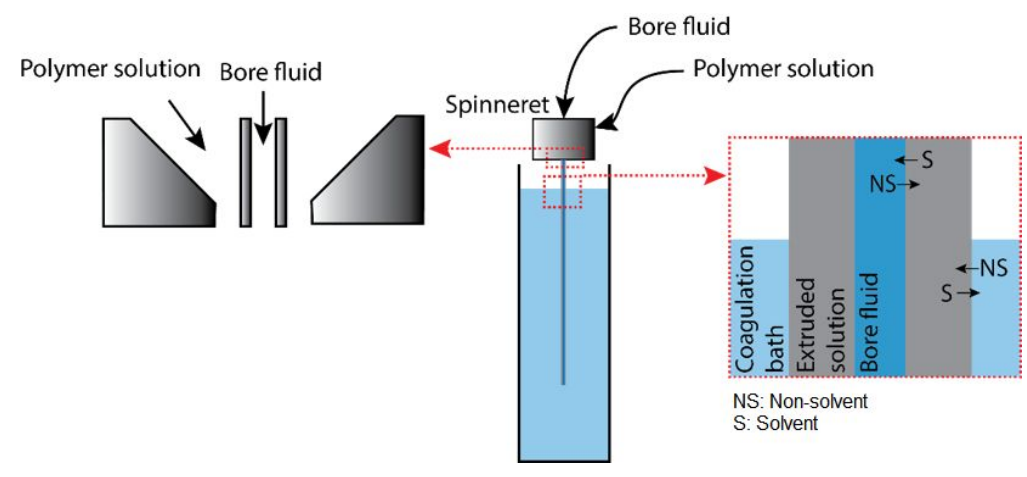

Figure S1. Schematic of Dry-jet wet spinning method for fabrication of hollow fiber membranes.

Table S1. Spinning conditions for the fabrication of hollow fiber membranes

\begin{tabular}{|c|c|}
\hline Parameter & Value \\
\hline Dope composition & 12 wt. \% PVDF/TEP \\
\hline Dope flow rate & $2.5 \mathrm{~mL} \mathrm{\textrm {min } ^ { - 1 }}$ \\
\hline Bore composition & $20 \mathrm{v} / \mathrm{v} \% \mathrm{TEP} /$ water \\
\hline Bore flow rate & $1.5 \mathrm{~mL} \mathrm{~min}^{-1}$ \\
\hline Coagulation bath & $30 \mathrm{v} / \mathrm{v} \% \mathrm{TEP} /$ water \\
\hline Air gap distance & $3 \mathrm{~cm}$ \\
\hline
\end{tabular}
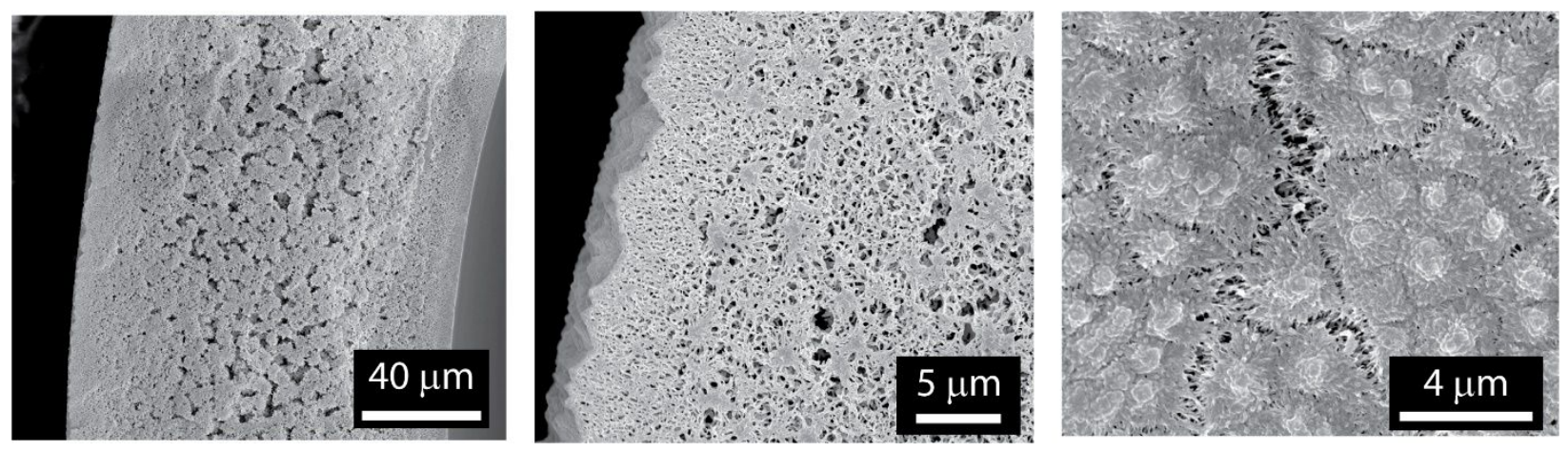

Figure S2. Cross-section and top-down SEM images of pristine PVDF hollow fiber membrane (support), fabricated through the dry-jet wet-spinning method. 


\section{Hollow Fiber Membrane Characterization}

Scanning electron microscopy (SEM) images were taken using a FEI Helios NanoLab 660 microscope. FTIR measurements were performed using the attenuated total reflection (ATR) module of the Bruker Alpha-p IR spectrometer. The FTIR measurements were performed using 24 high-resolution scans on each sample with a resolution of $4 \mathrm{~cm}^{-1}$. The gas permeation also was measured by a custom-built gas permeation setup (explained in Section S6). To characterize hollow fiber membranes in the DCMD, we first packed them in modules using two spacers fabricated through additive manufacturing (3-D printing). The detailed procedure for packing hollow fiber membranes and assembling modules can be found in Section S4. The DCMD performance for the control and omniphobic hollow fiber membranes was evaluated using a laboratory-scale direct contact membrane distillation (DCMD) unit illustrated in Figure S8.

\section{Initiated Chemical Vapor Deposition (iCVD)}

The initiated chemical vapor deposition (iCVD) of Polytetrafluoroethylene (PTFE) and poly $(1 \mathrm{H}, 1 \mathrm{H}, 2 \mathrm{H}, 2 \mathrm{H}$-perfluorodecyl acrylate) (PPFDA), were performed in a custom-made iCVD reactor with a total volume of $\sim 3600 \mathrm{~cm}^{3}$. In iCVD, as shown in Figure S3, the synthesis of polymers takes place in two key steps: (1) transport dynamics of reactants from the vapor phase to the substrate, and (2) polymerization reactions on the surface that lead to the polymerization on the surface. The properties of deposited polymers and thin films can be controlled by adjusting the polymerization reaction parameters (e.g., substrate and filament temperatures, and monomer-toinitiator ratio) and the vapor phase transport of species.

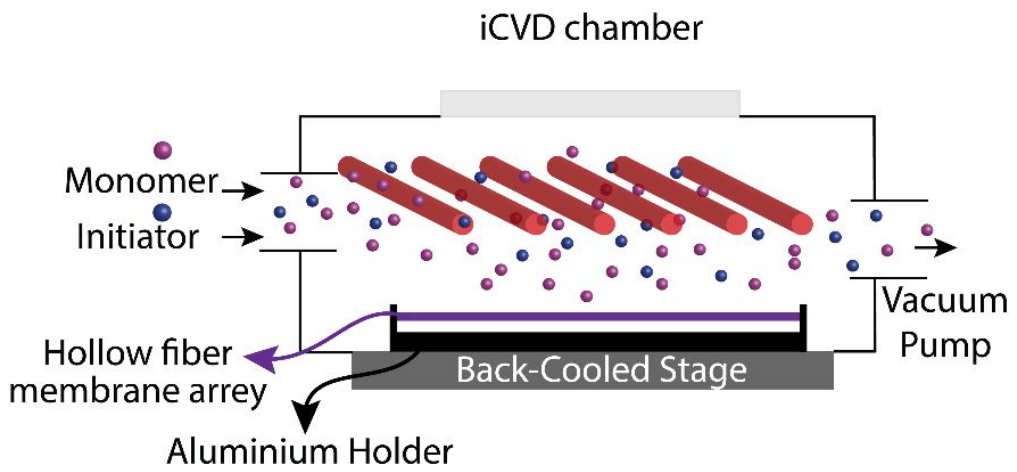

Figure S3. Schematic of the iCVD process used to assemble omniphobic structures on the hollow fiber membranes 


\section{Procedure for Making Omniphobic Hollow Fiber Membranes in iCVD}

To in situ polymerize PTFE and create the reentrant structures on the outer surface of PVDF hollow fiber membranes, we used Hexafluoropropylene oxide (HFPO) and Perfluorobutanesulfonyl fluoride (PBSF) as monomer and initiator, respectively. For this purpose, HFPO and PBSF were metered into the reactor using a mass flowmeter (MKS Instruments) and a precision needle valve (Swagelok) with flow rates of 12 and $1 \mathrm{sccm}$, respectively. The backside-cooled stage was maintained at $15^{\circ} \mathrm{C}$ and the Phosphor-Bronze filament (Good Fellow) -suspended $\sim 20 \mathrm{~mm}$ above the substrate- was heated to about $350^{\circ} \mathrm{C}$. A downstream throttle valve and a pressure controller (MKS Instruments) were connected to a dry vacuum pump (Edwards Vacuum) to maintain constant pressure at the setpoints (1200 mTorr). After five minutes, we terminated the deposition of PTFE by stopping the flow of the monomer and initiator, turning off the filament, and evacuating the reactor down to the base pressure ( $\sim 18 \mathrm{mTorr})$. At this point, to ensure we have a uniform PTFE coating on the outer surface of hollow fiber membranes, we filled the reactor with Argon (to bring it to the atmosphere), flipped the HFM holder over, and repeated the PTFE deposition at a similar condition for five minutes; then, and stopped the reaction as mentioned above.

Subsequently, within one minute, we started grafting PPFDA on the PTFE structures. To do so, PFDA and PBSF were metered into the iCVD reactor using precision needle valves (Swagelok). The flow rates of PFDA and PBSF were set to 0.26 and $1 \mathrm{sccm}$, respectively. The backside-cooled stage was maintained at $15{ }^{\circ} \mathrm{C}$, and the filament temperature was set at $350{ }^{\circ} \mathrm{C}$. We performed the iCVD of PPFDA for three minutes at a total pressure of 300 mTorr. In the end, we first stopped the monomer and allowed for the initiator to flow into the reactor for 30 seconds to terminate the growing polymer chains. Then, we turned off the filament, stopped the initiator flow, and evacuated the reactor down to the base pressure ( $\sim 18$ mTorr). Finally, to ensure the complete assembly of reentrant structures on the outer surface of hollow fiber membranes, we flipped the hollow fiber membrane holder and repeated the deposition from the beginning. After repeating the deposition process, the samples were left under vacuum for an additional 15 minutes to remove unreacted monomer and initiator molecules. After flipping the HFM holder over, we repeated the PPFDA deposition one more time for 3 minutes. 


\section{Section S2. Attenuated Total Reflectance Fourier Transformed Spectroscopy (ATR-FTIR)}

FTIR measurements were performed using the attenuated total reflection (ATR) module of the Bruker Alpha-p IR spectrometer. The ATR Diamond crystal was cleaned using isopropyl alcohol before every measurement. A small $(1 \mathrm{~cm})$ piece of the hollow fiber membrane sample was placed on the crystal of the ATR module. The spectra were collected with a resolution of $4 \mathrm{~cm}^{-1}$ and averaged over 24 scans.

Figure S4 compares the FTIR spectra for the PVDF hollow fiber membrane with the spectra of assembled PTFE and PPFDA layers. The characteristic peaks of PVDF, located at $766\left(\mathrm{CF}_{2}\right.$ bending) and $795\left(\mathrm{CH}_{2}\right.$ rocking $) \mathrm{cm}^{-1}$, assigned to the $\alpha$ phase of PVDF, ${ }^{1}$ can be seen in the spectra of omniphobic sample. Furthermore, we can identify a vibration at $1750 \mathrm{~cm}^{-1}$, associated with the stretching vibration of the ester groups of PPFDA. The peaks corresponding to the $\mathrm{CF}_{2}$ symmetric and asymmetric stretching vibration modes of PTFE, in the range of $1100-1200 \mathrm{~cm}^{-1}$, are the salient features in the spectra of the omniphobic sample.

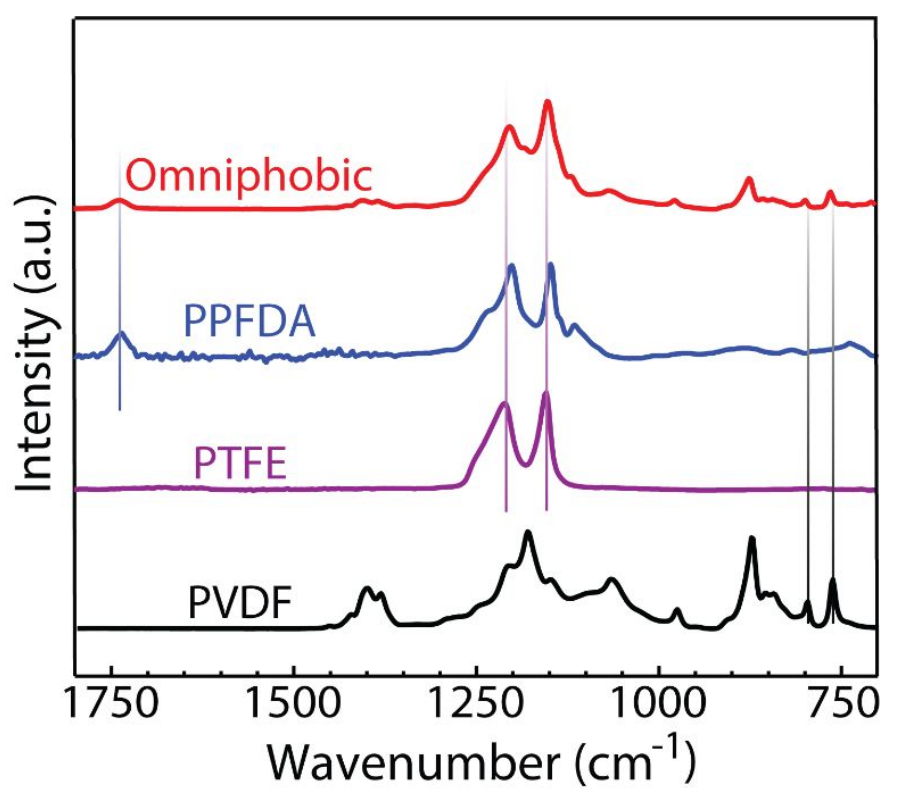

Figure S4. FTIR spectra of the pristine, coating materials, and omniphobic hollow fiber membranes. 


\section{Section S3. Contact Angle and Salt Scaling Analyses}

To measure the liquid contact angle on the membrane surfaces, we dipped them in the corresponding liquid and tracked the liquid upward (wetting) or downward (non-wetting) curves at the fiber-liquid interfaces using a high magnification digital camera (Opti-tekscope). For each liquid, the liquid contact angle value was measured by image analysis (ImageJ) on at least three different images. Figure S5 (a) shows the curvature of liquids in contact with dipped pristine and omniphobic hollow fiber membranes.

\section{Accelerated Salt Scaling Experiment}

The accelerated salt scaling experiment was performed to evaluate the scaling propensity of pristine and omniphobic hollow fiber membranes. For this reason, hollow fiber membranes were glued with epoxy to a glass slide and immersed in a $20 \mathrm{mM} \mathrm{CaCl}_{2}$ and $\mathrm{Na}_{2} \mathrm{SO}_{4}$ solution. The solution was capped, heated to $60{ }^{\circ} \mathrm{C}$ with stirring at $300 \mathrm{rpm}$ for 24 hours. Next, the hollow fiber membranes were removed from the solution and dried at room temperature $\left(\sim 22^{\circ} \mathrm{C}\right)$. Finally, the surfaces of the membranes were analyzed by SEM. Figures S5 (b) and (c) show the top-down SEM images of the pristine and omniphobic hollow fiber membrane surfaces. Also, Figure S6 shows lower magnification SEM images that were processed in ImageJ to find the area fraction of salt crystals on pristine and omniphobic hollow fiber membranes. 


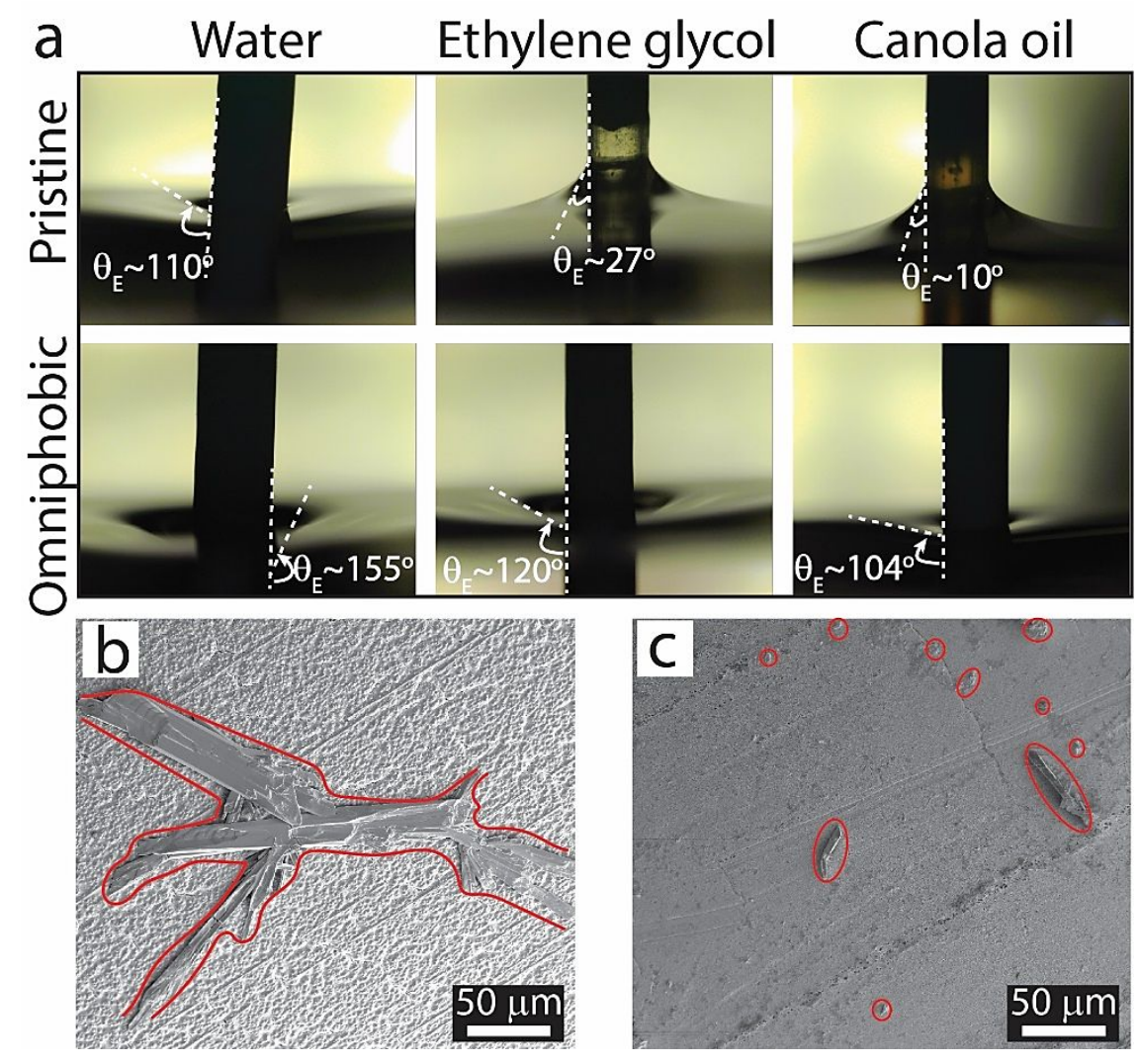

Figure S5. (a) Digital microscope optical images of the curvature of different liquids in contact with pristine and omniphobic hollow fiber membranes dipped in water, ethylene glycol, and canola oil. The annotations show the approximate contact angles measured using image analysis (ImageJ). (b \& c) SEM images of outer surfaces of pristine and omniphobic hollow fiber membrane after accelerated salt scaling experiment. The salt crystals on membranes have been inscribed with a red line.
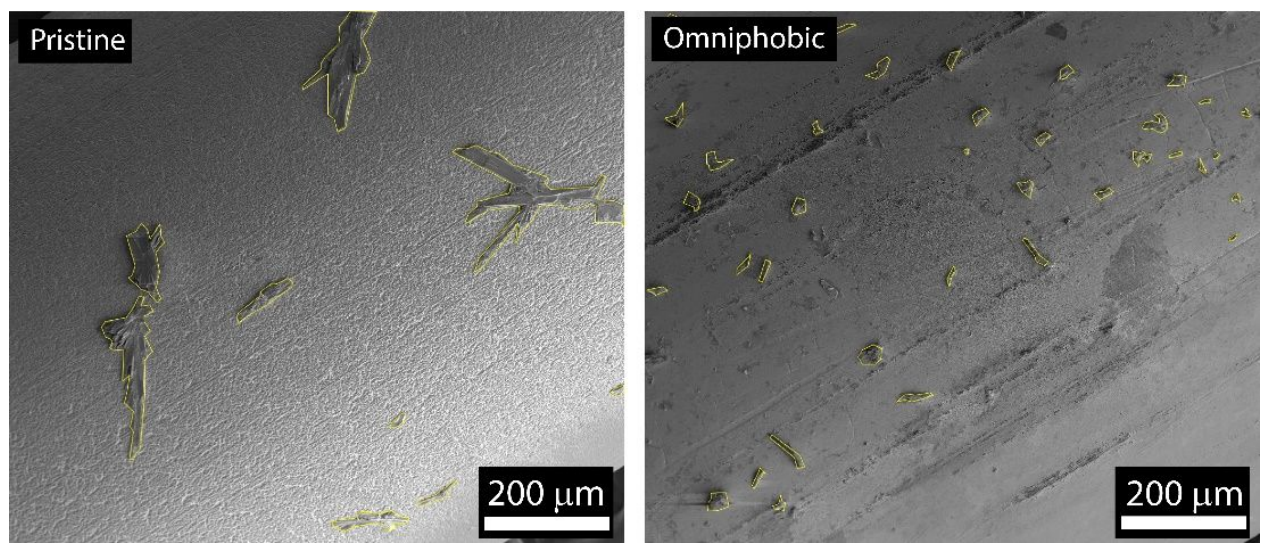
Figure S6. A sample of image analysis (ImageJ) used to measure the area fraction of salt on the outer surface of pristine and omniphobic hollow fiber membranes. Salt crystals were ascribed manually at high magnification, and then the area analysis was performed to measure the total area of inscribed regions.

Table S2. The average values of contact angle for water, ethylene glycol, and canola oil, and area fraction of salt crystals on pristine and omniphobic hollow fiber membrane surfaces.

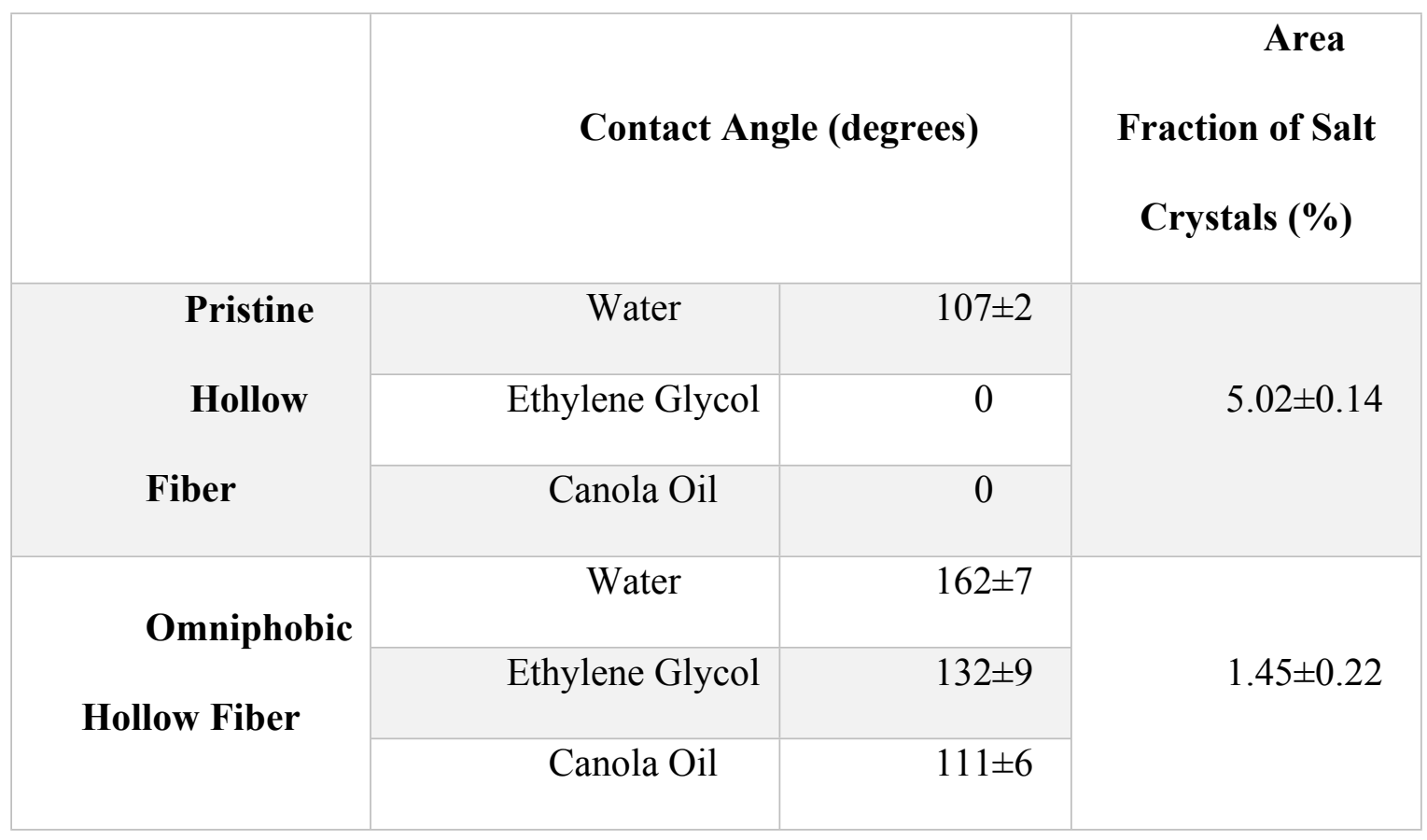

\section{Section S4. Assembling and Testing Modules with Organized Packing}

To assemble the modules, we adopted our optimal simulation results published elsewhere. ${ }^{2}$ As shown in Figure 3, in main text, after passing fibers from the 3D printed spacers and placing them in the polycarbonate tubular housing $(\mathrm{L}=10 \mathrm{~cm})$, we used epoxy to glue the two ends of the module. Figure 3 (a), in the main text, displays the schematic of 3-D printed spacers. Also, the procedure for assembling the modules with organized packing is illustrated in Figure 3 (b), in the main text. As shown, the assembling process includes four stages. (b-1): passing hollow fiber membranes through the 3D printed spacers and sealing one end with epoxy. (b-2): sliding the other spacer 
away. (b-3): inserting the spacers and hollow fiber membrane bundle into a polycarbonate tube with an inner diameter of $9.525 \mathrm{~mm}$, and sealing both ends with epoxy. (b-4): image of the final version of the assembled module with organized packing.

In addition, we fabricated modules with different packing densities by changing the spacer design, shown in Figure S7 (a). Compared to Module 2 (shell diameter $19 \mathrm{~mm}$ ), Module 1 (shell diameter $\sim 9.525 \mathrm{~mm}$,) was fabricated using smaller spacers and shells but with the same number of fibers.

The packing density of Modules 1 and 2 was $21 \%$ and 5\%, respectively. Figure 7 (b) shows that, when the water production is normalized to the volume, the reduction in tube volume led to $80 \%$ enhancement of water production rate per volume of the module when the feed temperature was set at $60{ }^{\circ} \mathrm{C}$.
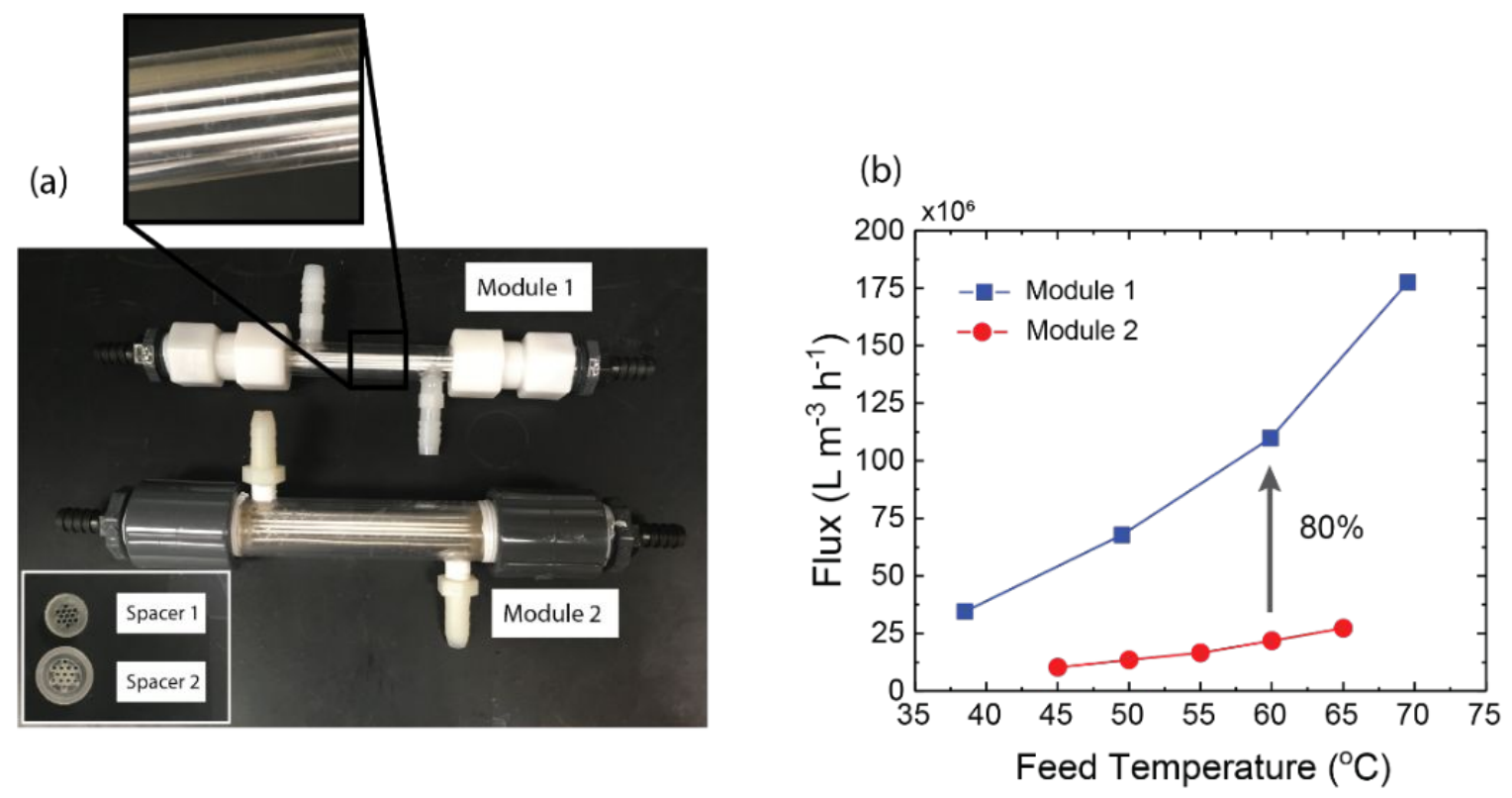

Figure S7. (a) Two prototype modules fabricated with different spacers. The inset image shows the size of the spacers used for each module. The magnified image demonstrates the spacing between fibers. (b) Water flux normalized to the volume of the module as a function of feed temperature for Module 1 and Module 2. 


\section{Direct Contact Membrane Distillation (DCMD) setup and tests}

The performance of the control and omniphobic hollow fiber membranes was evaluated using a laboratory-scale direct contact membrane distillation (DCMD) unit illustrated in Figure S8. The water vapor flux across the membrane was determined by the gravimetric method. ${ }^{3}$ The temperatures of the feed and permeate streams at the entrance of the module were measured to be $68^{\circ} \mathrm{C}$ and $18^{\circ} \mathrm{C}$, respectively. To calculate the salt rejection by tracking the $\mathrm{NaCl}$ concentration change in the permeate stream, we continuously measured the distillate ionic conductivity using a conductivity probe (Oakton 2700 , Cole Parmer). ${ }^{4}$ The fresh (control) feed solution was composed of 0.6 M sodium chloride in DI water. Where needed, sodium dodecyl sulfate (SDS) was added to the feed solution to reduce the surface tension of the feed solution.

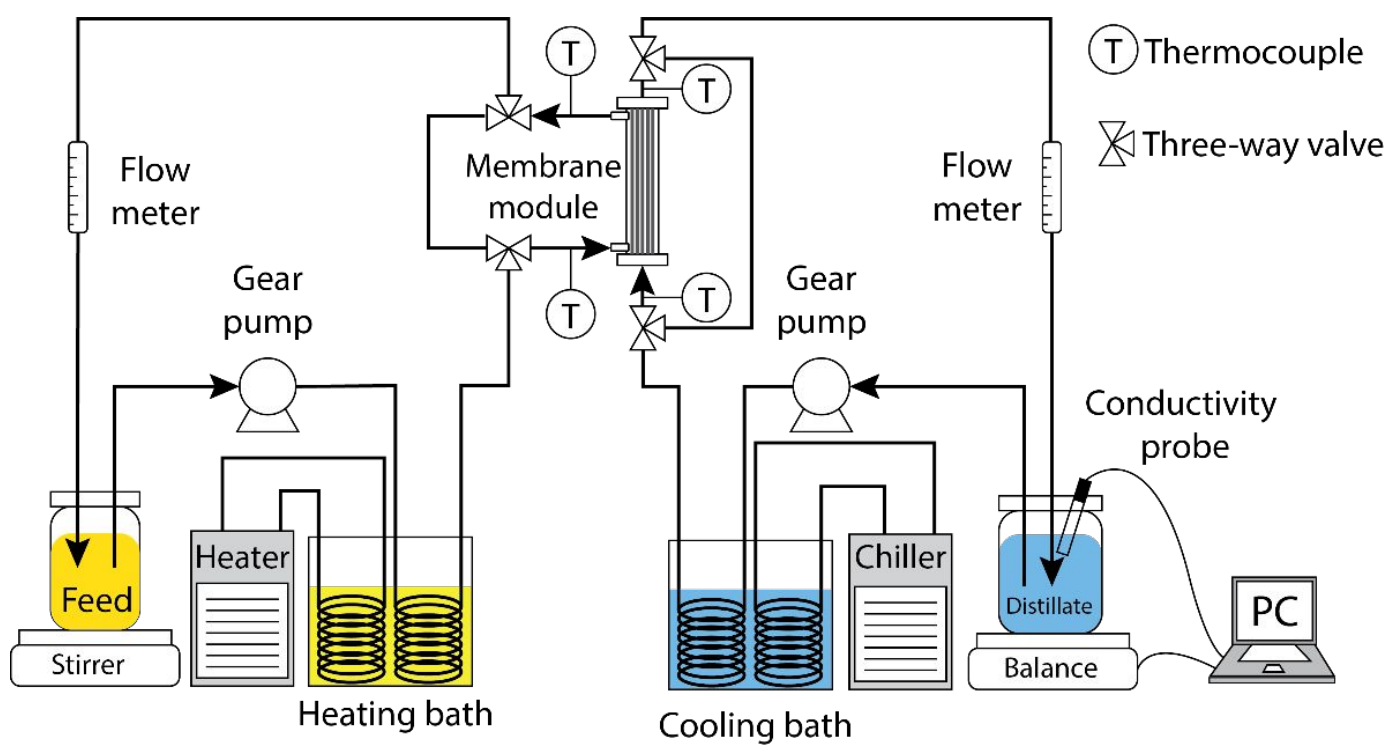

Figure S8. Schematic diagram of the laboratory-scale DCMD experimental setup.

To confirm our previous modeling results, ${ }^{2}$ we assembled randomly-packed hollow fiber membrane modules and compared the result with that of organized packings. Figure S9 (a) indicates that, on average, organized hollow fiber membrane packing provides ca. $12 \%$ more permeate flux. This enhancement can be attributed to the better thermal efficiency of the organized packing modules, shown in Figure S9 (b); similar observations also have been shown in previous modeling studies. ${ }^{2,5}$ 

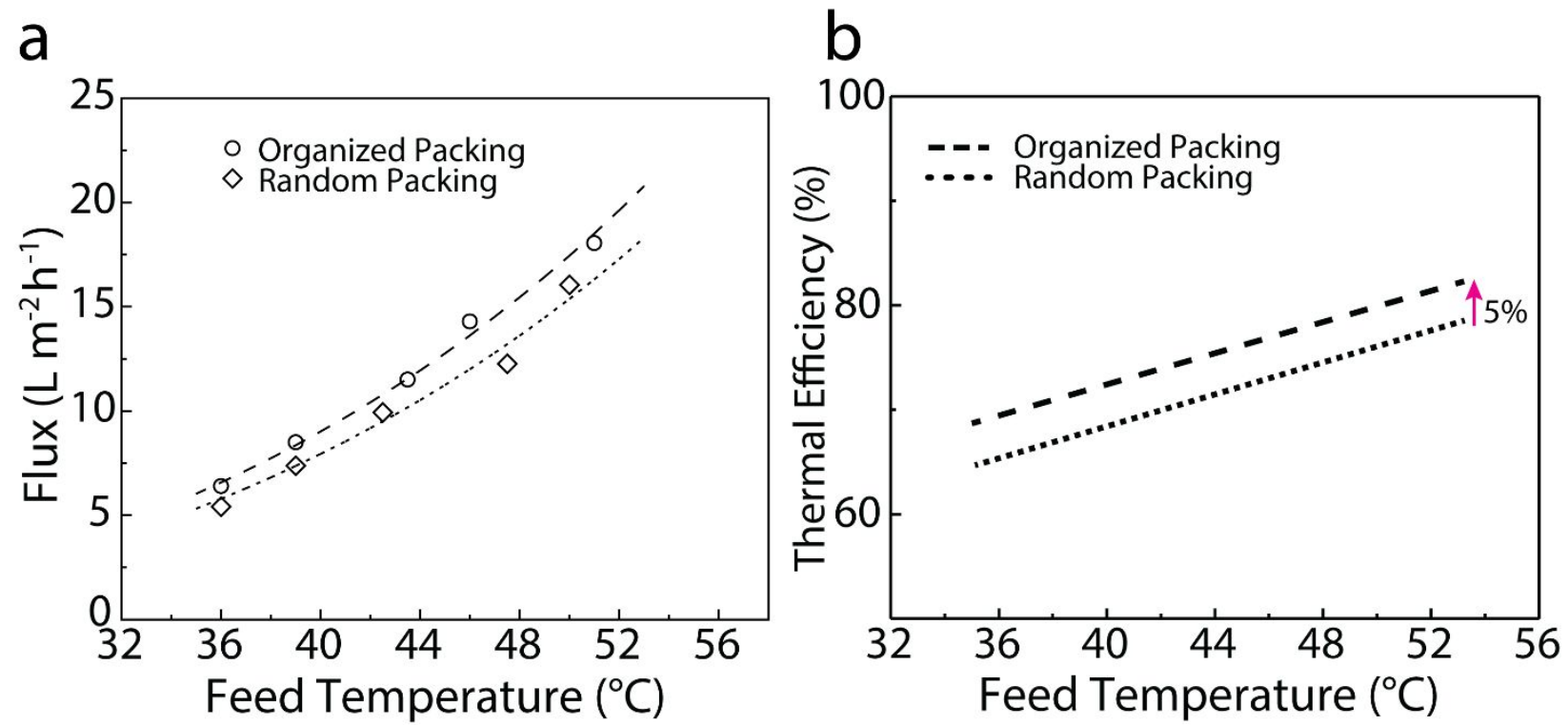

Figure S9. (a \& b) Performance (flux and thermal efficiency) comparison of an organized hollow fiber membrane packing with a randomly packed one. Here, for the organized modules, the R/a parameter was $0.3 .^{2}$ The packing density of modules was $15.4 \%$.

Table S3. List of some commercial hollow fiber membranes

\begin{tabular}{|llll|}
\hline ID & Membrane material & Vendor & Reference \\
\hline A & PVDF & N.A. & 6 \\
B & PTFE & Markel Corp., Plymouth Meeting, PA, USA & 7 \\
C & PP & Microdyn-Nadir, Germany & 8 \\
D & PP & Membrana, Germany ${ }^{\mathrm{a}}$ & 9 \\
E & PP & Hyflux, Singapore & 10 \\
\hline
\end{tabular}

a: now 3M separation and purification division, Germany

\section{Section S5. Mathematical Modeling of Organized Hollow Fiber Membrane Packings}

We solved coupled mass, momentum, and energy phenomena taking place in the DCMD process using COMSOL Multiphysics 5.2a installed on one computing node including 16 cores (3.2 GHz) and 128 GB RAM. The computational domain includes the symmetrical part of a hollow fiber membrane in the control volume of the shell. Figure S10 shows a unit cell of close-packed configuration lattice geometry matched to the experimental module configuration. The shaded region is the symmetrical computational domain considered to perform the simulations. The detailed simulation approach and governing equations can be found in our previous work 
(Mohammadi Ghaleni et al., 20182). Table S4 also presents the operating and physical parameters we used in the simulations.

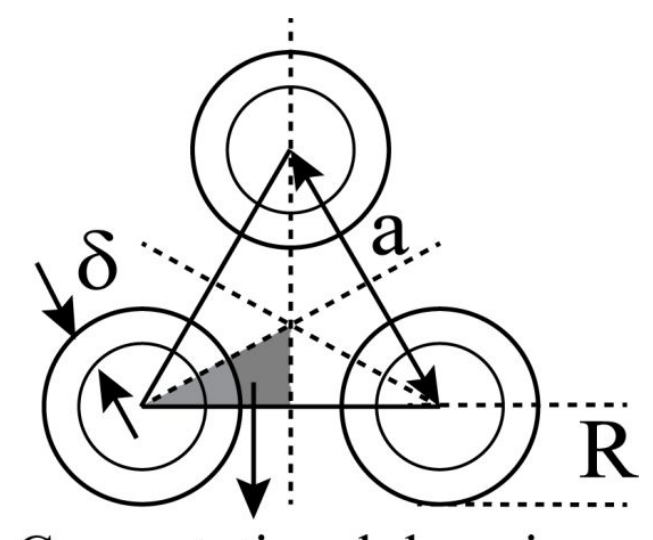

\section{Computational domain}

Figure S10. The computational domain used to perform the simulations. ${ }^{2}$

Table S4. The parameters used to perform the DCMD simulations

\begin{tabular}{|c|c|c|c|}
\hline Parameter & Value & Parameter & Value \\
\hline Feed temperature & $323-343 \mathrm{~K}$ & Pore diameter $\left(\mathrm{d}_{\mathrm{p}}\right)$ & $0.2 \mu \mathrm{m}$ \\
\hline Distillate temperature & $293-298 \mathrm{~K}$ & Tortuosity $(\tau)$ & 2.5 \\
\hline Feed velocity & $0.1-1 \mathrm{~m} / \mathrm{s}$ & Porosity $(\varepsilon)$ & 0.72 \\
\hline Distillate velocity & $0.1-1 \mathrm{~m} / \mathrm{s}$ & Fiber length $(\mathrm{L})$ & $10 \mathrm{~cm}$ \\
\hline Fiber outer radius $(\mathrm{R})$ & $500-600 \mu \mathrm{m}$ & Feed salinity & $35 \mathrm{~g} / 1$ \\
\hline Fiber thickness $(\delta)$ & $350-450 \mu \mathrm{m}$ & $\mathrm{R} / \mathrm{a}$ & $0.3-0.4$ \\
\hline
\end{tabular}

\section{Section S6. Size Distribution of Oil Droplets in the Feed Solution}

We prepared a synthetic oil emulsion that simulates oilfield-produced water to test the anti-oilfouling properties of the membranes. Figure 11 (a) shows that the emulsions were foggy compared to pure water. We did not observe any phase separation on the emulsion after 48 hours of relaxing the solution, which indicates that the emulsions were stabilized with the $0.07 \mathrm{mM}$ SDS. Using image analysis on the micrographs of the emulsions, Figures S11 (b) \& (c), we obtained the histogram of the droplet size distribution. The histograms show that the distribution of droplet size is wider in $500 \mathrm{ppm}$ oil concentration. However, the mean droplet size was relatively similar $(\sim 5$ 
$\mu \mathrm{m}$ ). The largest droplet size was $\sim 23 \mu \mathrm{m}$ for $500 \mathrm{ppm}$, compared to $\sim 12 \mu \mathrm{m}$ for $100 \mathrm{ppm}$. Oilfieldproduced water typically contains $2-565 \mathrm{ppm}^{14,6,11}$ with oil droplet size in the range of 2 to $30 \mu \mathrm{m} .{ }^{6}$ Hence, we made our synthetic feed emulsion using $500 \mathrm{ppm}$ oil in water and simulated the higher limit of oilfield produced water resources.

(a)

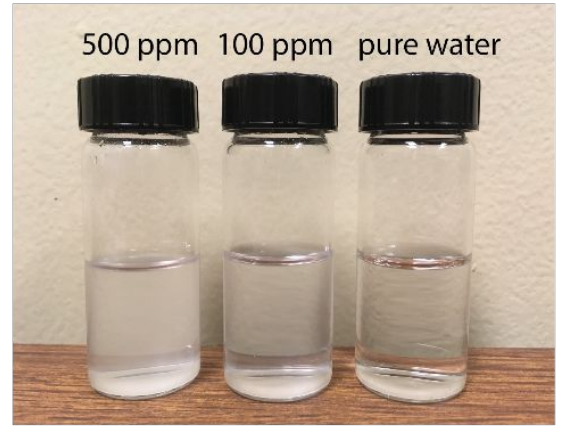

(b)

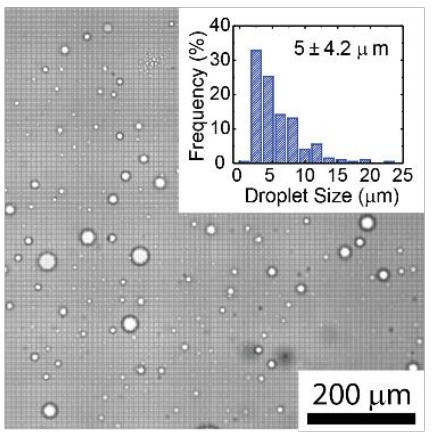

(c)

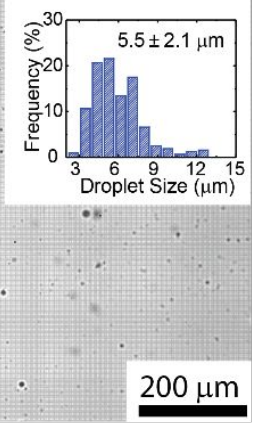

Figure S11. (a) Samples of $500 \mathrm{ppm}, 100 \mathrm{ppm}$ oil, and pure water. Optical microscopic image of (b) $500 \mathrm{ppm}$ oil emulsion and (c) $100 \mathrm{ppm}$ oil emulsion. The insets in (b) and (c) display the size of oil droplets using image analysis on the micrographs.

\section{Section S7. Gas Permeation Measurements}

The gas permeation was measured by a custom-built gas permeation setup using nitrogen gas, as illustrated in Figure S12. The measurement procedure has been reported. ${ }^{12}$ The gas permeability coefficient $\left(\mathrm{B}_{\mathrm{g}}\right)$ of membranes was calculated using equation $(\mathrm{S}-1):^{13}$

$$
\text { Nitrogen Flux }=B_{g} \Delta P
$$

where $\Delta P(\mathrm{kPa})$ is the pressure difference across the membrane. 


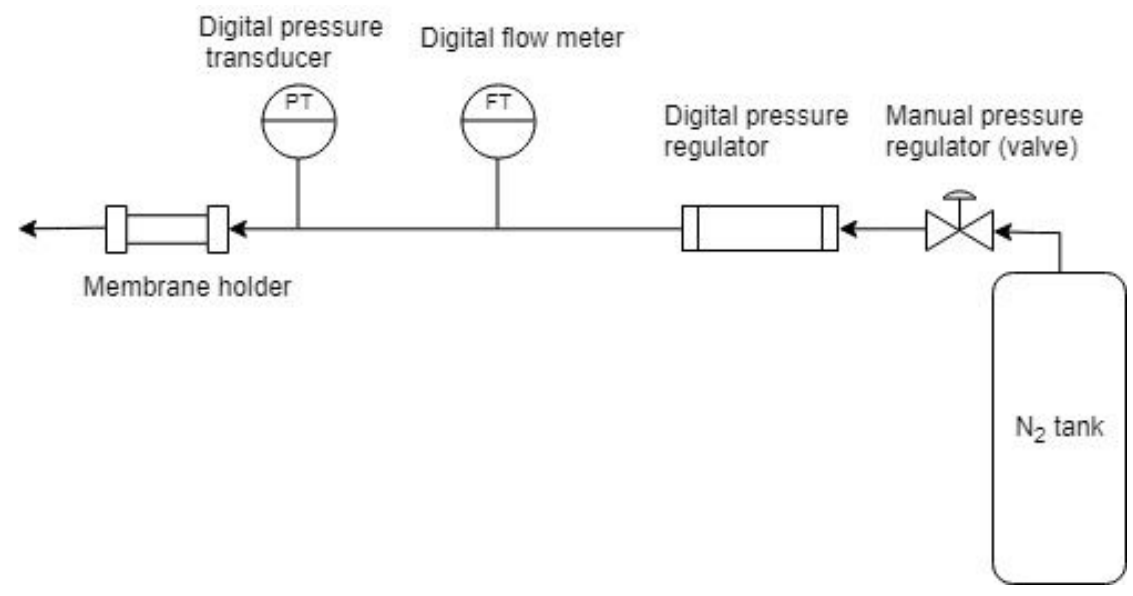

Figure S12. Schematic of gas permeation setup.

Figure S13 shows the gas permeation data for the pristine and omniphobic hollow fiber membranes. As shown, after assembling PTFE reentrant structures and subsequent coating with PPFDA, the gas permeation dropped by an average of $40 \%$. The reduction results because the surface porosity of PVDF support declines after making them omniphobic.

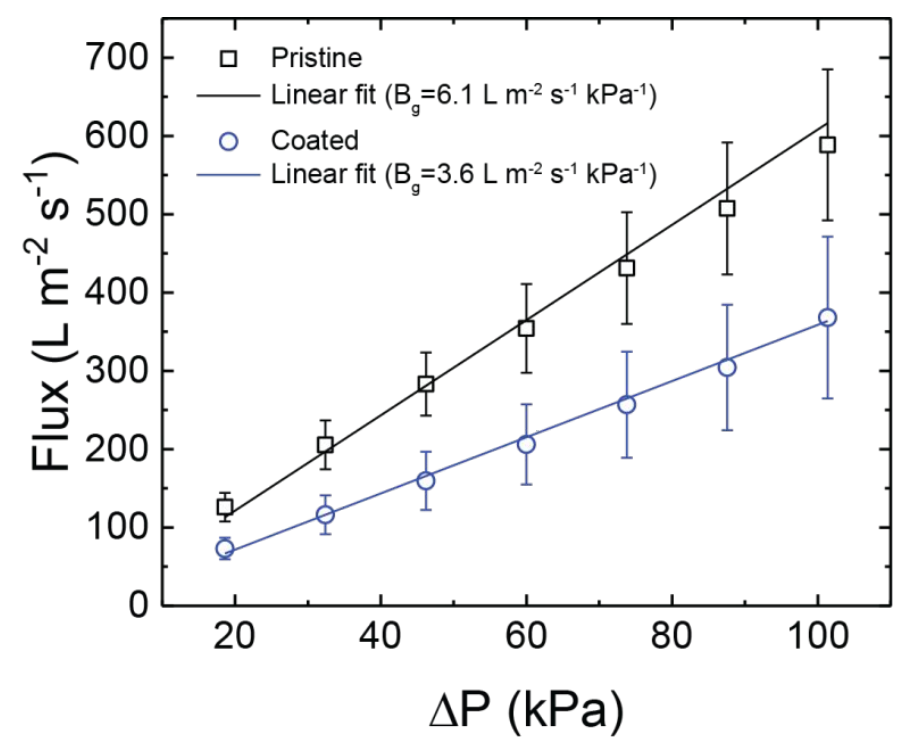

Figure S13. Gas permeation of the pristine and coated hollow fiber membrane. The correlation factors, $\mathrm{R}^{2}$, for the pristine and coated hollow fiber membrane were 0.9966 and 0.9972 , respectively. 


\section{Pore size Distribution Before and After Coating}

To provide additional information about the changes in surface porosity of hollow fiber membranes after coating, we performed image analysis on a few different spots of the omniphobic and pristine samples. Figure S14 represents the approach we took to evaluate the pore size distribution on the outer surface of the hollow fiber membranes. From our analysis, we concluded that the pore size distribution shifts slightly towards smaller pore sizes after coating.

(a) Pristine

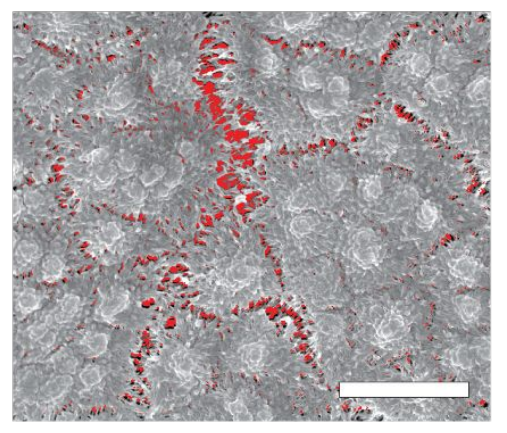

(b) Coated

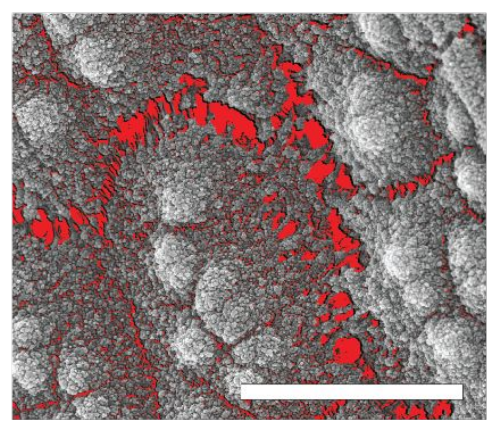

(c)

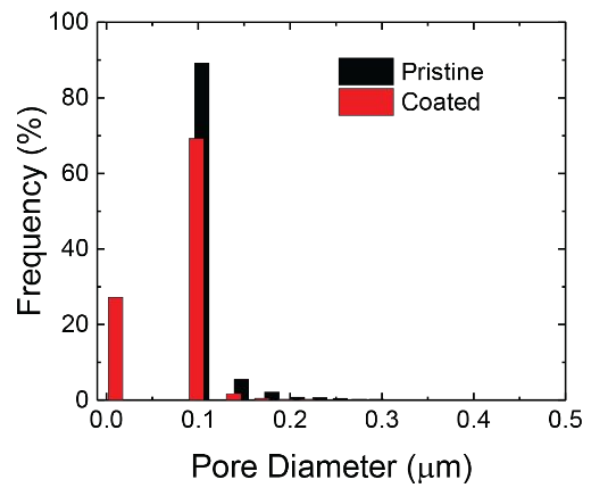

Figure S14. SEM image of (a) pristine and (b) omniphobic hollow fiber membrane. Scale bars in (a) and (b) are 4 and $5 \mu \mathrm{m}$. (c) pore size distribution using image analysis on (a) and (b). The mean pore sizes for the pristine and coated hollow fiber membrane were 0.11 and $0.077 \mu \mathrm{m}$, respectively.

\section{References}

(1) Gregorio, Jr., R.; Ueno, E. M. Effect of Crystalline Phase, Orientation and Temperature on the Dielectric Properties of Poly (Vinylidene Fluoride) (PVDF). J. Mater. Sci. 1999, 34, 4489-4500.

(2) Mohammadi Ghaleni, M.; Bavarian, M.; Nejati, S. Model-Guided Design of HighPerformance Membrane Distillation Modules for Water Desalination. J. Memb. Sci. 2018, $563,794-803$.

(3) Nejati, S.; Boo, C.; Osuji, C. O.; Elimelech, M. Engineering Flat Sheet Microporous PVDF Films for Membrane Distillation. J. Memb. Sci. 2015, 492, 355-363.

(4) Boo, C.; Lee, J.; Elimelech, M. Omniphobic Polyvinylidene Fluoride (PVDF) Membrane 
for Desalination of Shale Gas Produced Water by Membrane Distillation. Environ. Sci. Technol. 2016, 50, 12275-12282.

(5) Deshmukh, A.; Elimelech, M. Understanding the Impact of Membrane Properties and Transport Phenomena on Energetic Performance of Membrane Distillation Desalination. $J$. Memb. Sci. 2017.

(6) Chew, N. G. P.; Zhao, S.; Loh, C. H.; Permogorov, N.; Wang, R. Surfactant Effects on Water Recovery from Produced Water via Direct-Contact Membrane Distillation. J. Memb. Sci. 2017, 528, 126-134.

(7) Huang, F. Y. C.; Arning, A. Performance Comparison between Polyvinylidene Fluoride and Polytetrafluoroethylene Hollow Fiber Membranes for Direct Contact Membrane Distillation. Membranes (Basel). 2019, 9, 52.

(8) Al-Obaidani, S.; Curcio, E.; Macedonio, F.; Di Profio, G.; Al-Hinai, H.; Drioli, E. Potential of Membrane Distillation in Seawater Desalination: Thermal Efficiency, Sensitivity Study and Cost Estimation. J. Memb. Sci. 2008, 323, 85-98.

(9) Drioli, E.; Ali, A.; Simone, S.; MacEdonio, F.; Al-Jlil, S. A.; Al Shabonah, F. S.; Al-Romaih, H. S.; Al-Harbi, O.; Figoli, A.; Criscuoli, A. Novel PVDF Hollow Fiber Membranes for Vacuum and Direct Contact Membrane Distillation Applications. Sep. Purif. Technol. 2013, 115, 27-38.

(10) Teoh, M. M.; Bonyadi, S.; Chung, T.-S. S. Investigation of Different Hollow Fiber Module Designs for Flux Enhancement in the Membrane Distillation Process. J. Memb. Sci. 2008, $311,371-379$.

(11) Fakhru'l-Razi, A.; Pendashteh, A.; Abdullah, L. C.; Biak, D. R. A.; Madaeni, S. S.; Abidin, Z. Z. Review of Technologies for Oil and Gas Produced Water Treatment. J. Hazard. Mater. 2009, 170, 530-551.

(12) Mohammadi Ghaleni, M.; Al Balushi, A.; Kaviani, S.; Tavakoli, E.; Bavarian, M.; Nejati, S. Fabrication of Janus Membranes for Desalination of Oil-Contaminated Saline Water. ACS Appl. Mater. Interfaces 2018, acsami.8b16621.

(13) Souhaimi, M. K.; Matsuura, T. Membrane Distillation: Principles and Applications; Elsevier, 2011. 
S-18 